\title{
Searching for pair production of SUSY particle in leptonic final states at the CMS
}

\author{
Ashraf Kasem on behalf of the CMS Collaboration ${ }^{a, b, *}$ \\ ${ }^{a}$ Deutsches Elektronen Synchrotron, \\ Notkestraße 85, D-22603 Hamburg, Germany \\ ${ }^{b}$ RWTH Aachen University, III. Physikalisches Institut A, \\ Otto-Blumenthal-Str., 52056 Aachen, Germany \\ E-mail: ashraf.mohamed@cern.ch
}

\begin{abstract}
Search for new physics beyond the standard model in final states with two opposite-sign sameflavor leptons and missing transverse momentum or at least two jets and two isolated same-sign or three or more charged leptons are presented. This search is done using data corresponding to a sample of proton-proton collisions at $\sqrt{s}=13 \mathrm{TeV}$ with an integrated luminosity of $137 \mathrm{fb}^{-1}$, collected by the CMS experiment at the LHC. The numbers of observed events are consistent with the expectations from standard model processes. The results are used to set lower mass limits on various supersymmetric particles at a $95 \%$ confidence level.
\end{abstract}

40th International Conference on High Energy physics - ICHEP2020

July 28 - August 6, 2020

Prague, Czech Republic (virtual meeting)

\footnotetext{
${ }^{*}$ Speaker
} 


\section{Introduction}

In this proceeding, two recent searches for supersymmetry (SUSY) in different leptonic final states are documented. The first search looks at final state with two opposite-sign (OS), same-flavor (SF) light leptons (electrons $e$, or muons $\mu$ ), referred to as OSSF leptons and missing transverse momentum ( $\left.p_{\mathrm{T}}^{\mathrm{miss}}\right)$, assuming $R$-parity conserving SUSY models. The second search is done in final states with at least one pair of same-sign (SS) leptons, considering both $R$-parity conserving and $R$-parity violating SUSY models. The $R$-parity conservation leads to the stability of the Lightest Supersymmetric Particle (LSP), which escapes detection. The LSP signature is an imbalance in the measured transverse momentum of which the magnitude depends on the particle mass. In contrast, the $R$-parity violation allows the LSP to decay into SM particles only, leading to various signatures with small or no missing transverse momentum. Both searches, the OSSF and SS, are done using datasets of proton-proton (pp) collisions at $\sqrt{s}=13 \mathrm{TeV}$ recorded by the CMS detector [1] at the CERN LHC in 2016-2018, corresponding to an integrated luminosity of $137 \mathrm{fb}^{-1}$.

\section{Search for opposite-sign same-flavor leptons}

This search considers SUSY models where the final state leptons are produced from the decay of an on- or off-shell $Z$ boson or from an slepton ( $\widetilde{\ell}$, the SUSY partners of SM leptons). In the case of leptons from an off-shell $Z$ boson, we consider a model at which the $m_{\ell \ell}$ distribution makes an edge-like shape [2] and another model with leptons from an slepton decay making no resonance around the $Z$ boson mass. In the case of leptons from on-shell $Z$ boson, the expected signature is an excess of events with a dilepton invariant mass $\left(\mathrm{m}_{\ell \ell}\right)$ around the mass of the $Z$ boson. Figure 1 shows the diagrams for the signals considered in this proceeding [3].
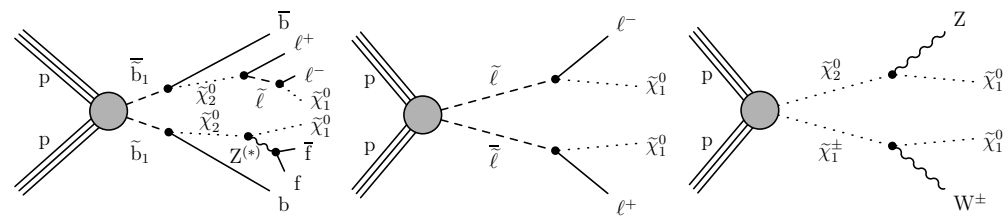

Figure 1: Diagrams for models of b-squark ( $\left.\widetilde{\mathrm{b}_{1}}\right)$ pair production (left), direct slepton pair production (middle), and neutralino/chargino $\left(\widetilde{\chi}_{1}^{0} \widetilde{\chi}_{1}^{ \pm}\right)$EWK production (right) [3].

The analysis applies common selections for all of the signals considered in this search. An OS lepton pair where the leading lepton is required to have $p_{T}>25 \mathrm{GeV}$, the next-to-leading lepton is required to have $p_{T}>10 \mathrm{GeV}$, and both leptons are required to be within $|\eta|<2.4$. The dilepton system is required to have $p_{T}^{\ell \ell}>50 \mathrm{GeV}$ and $m_{\ell \ell}>20 \mathrm{GeV}$. Additionally, in order to suppress the backgrounds with instrumental $p_{\mathrm{T}}^{\text {miss }}$ the angle azimuthal angle between the $\vec{p}_{\mathrm{T}}^{\text {miss }}$ and the two highest- $p_{T}$ jets $\left(\Delta \phi\left(\vec{p}_{T}^{j_{1,2}}, \vec{p}_{\mathrm{T}}^{\text {miss }}\right)\right)$ is required to be $>0.4$.

The main backgrounds for this search are considered to be from three sources. The first set of backgrounds are those from flavor-symmetric processes $\left(t \bar{t}, W W, \mathrm{t} W\right.$, and $\left.Z / \gamma^{*} \rightarrow \tau^{+} \tau^{-}\right)$. Since the Different Flavor (DF) and SF events are produced at the same rate. These backgrounds are estimated from data using Control Regions (CRs) with events passing the same selections as the 
Search Regions (SRs) but inverting the flavor-symmetry criteria, i.e. requiring DF leptons. A transfer factor, $R_{\mathrm{SF} / \mathrm{DF}}$, is used to estimate the background and absorb the differences in reconstruction and identification efficiencies between the SF and DF events.

The second set of backgrounds are those from events with significant instrumental $p_{\mathrm{T}}^{\text {miss }}$ dominated by Drell-Yan+jets (DY+jets). Although the DY+jets events do not have a $p_{\mathrm{T}}^{\text {miss }}$ source, it enters the SRs due to the instrumental $p_{\mathrm{T}}^{\text {miss }}$, which arises from mismeasurements of the $\vec{p}_{T}$ of the reconstructed objects. This background is estimated using the so-called $p_{\mathrm{T}}^{\text {miss }}$ "template" method [4-7] at which the $p_{\mathrm{T}}^{\text {miss }}$ distribution in DY+jets events is estimated directly from $\gamma+\mathrm{jets}$ data, while the normalization is taken from a region with $50<p_{\mathrm{T}}^{\text {miss }}<100 \mathrm{GeV}$.

The third set of backgrounds are those from events with a leptonically decaying $Z$ boson and neutrinos $\left(Z / \gamma^{*}+v, W Z\right.$, and $\left.t \bar{t} Z\right)$, which are characterized by large $p_{\mathrm{T}}^{\text {miss }}$ values. The shape for these backgrounds are estimated directly from simulation, and the normalization is determined in trileptons and two pairs of OSSF leptons data CRs.

For the edge-like signal, the background events are mainly from $t \bar{t}$. The SR is defined with two different methods. The first method defines a Bayesian likelihood discriminator to classify the events into $t \bar{t}$-like or and non- $t \bar{t}$-like, exploiting different kinematic properties of $t \bar{t}$ events compared with the signal events. The resulting probability distribution is split further into bins based on the $\mathrm{m}_{\ell \ell}$ and $n_{\mathrm{b} \text {-jet }}$. Figure 2 (left) shows the resulting distribution of the $n_{\mathrm{b} \text {-jet }} \geq 1 \mathrm{SR}$. The second method uses a fit to the dilepton mass distribution. The fit result is shown in Fig. 2 (middle). A best-fit signal yield of $27 \pm 22$ events is obtained when evaluating the signal hypothesis in the edge fit SR with a fitted edge position of $\mathrm{m}_{\ell \ell}=294_{-20}^{+12} \mathrm{GeV}$. The results are used to set upper limits at 95\% CL on the production cross-section as a function of $\widetilde{\chi}_{2}^{0}$ and $\widetilde{b_{1}}$ masses is shown in Fig. 3 (left). The search excludes $m_{\widetilde{\mathrm{b}_{1}}}$ mass up to $1400 \mathrm{GeV}$ [3].

For slepton direct production signals, the sensitivity is driven by combining multiple search bins. This analysis considers events with jets coming mainly from Initial State Radiations (ISR). The $p_{\mathrm{T}}^{\text {miss }}$ distribution of data events in the slepton SRs is shown together with the SM background predictions in Fig. 2 (right). The observed data yields are consistent with the SM predictions. The results are used to set upper limits at $95 \% \mathrm{CL}$ on the signal production cross-section as shown in Fig. 3 (middle). The search excludes slepton mass up to $650 \mathrm{GeV}$ [3].

In the case of electroweak production of an on-shell $Z$ boson, the sensitivity is driven by binning the events in the $Z$ boson mass window. The binning is done using several variables such as $n_{\text {jets }}, n_{\text {b-jets }}$, etc. The $W$ boson decays hadronically, for which two jet cone sizes are used 0.8 and 0.4 . These two cone sizes are used to account for the boosted and resolved $W$ boson decay products. For this signal, the observed data yields are consistent with the SM background predictions. More results in this regard are shown in this Ref. [3]. The results are used to set a cross-section upper limits and the exclusion contours at $95 \%$ Confidence Level (CL) as a function of the $\widetilde{\chi}_{1}^{ \pm} / \widetilde{\chi}_{2}^{0}$ and $\widetilde{\chi}_{1}^{0}$ masses. The search excludes $\widetilde{\chi}_{1}^{ \pm} / \widetilde{\chi}_{2}^{0}$ masses up to $750 \mathrm{GeV}$ [3], as shown in Fig. 3 (right).

\section{Search for two same-sign or three or more leptons}

The set of SUSY models studied in this search are those with conserved or violated $R$-parity. The signals studied in this analysis are those shown in Fig. 4. In general, pair-produced gluinos or 

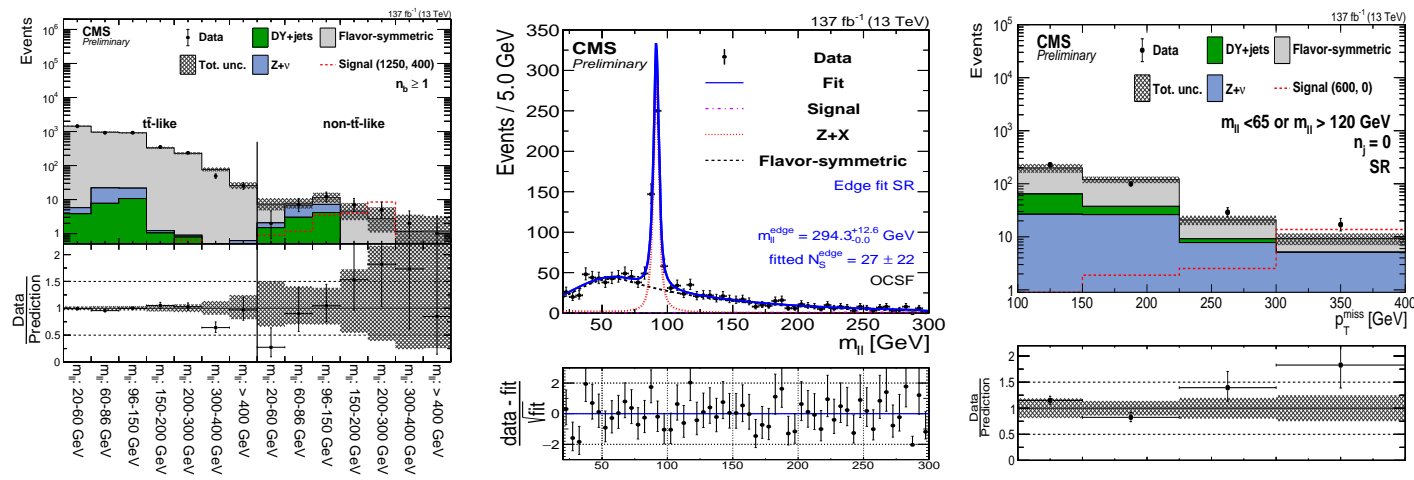

Figure 2: Left: results of the counting experiment in the edge search regions. Middle: fit to data of the dilepton mass $\left(\mathrm{m}_{\ell \ell}\right)$ distributions in the edge fit search regions, under the signal+background hypothesis, projected onto the SF data samples [3]. Right: Distribution of $p_{\mathrm{T}}^{\text {miss }}$ for events in the slepton for regions without jets for slepton signal mass of $600 \mathrm{GeV}$ and a massless $\widetilde{\chi}_{1}^{0}$ particle [3].
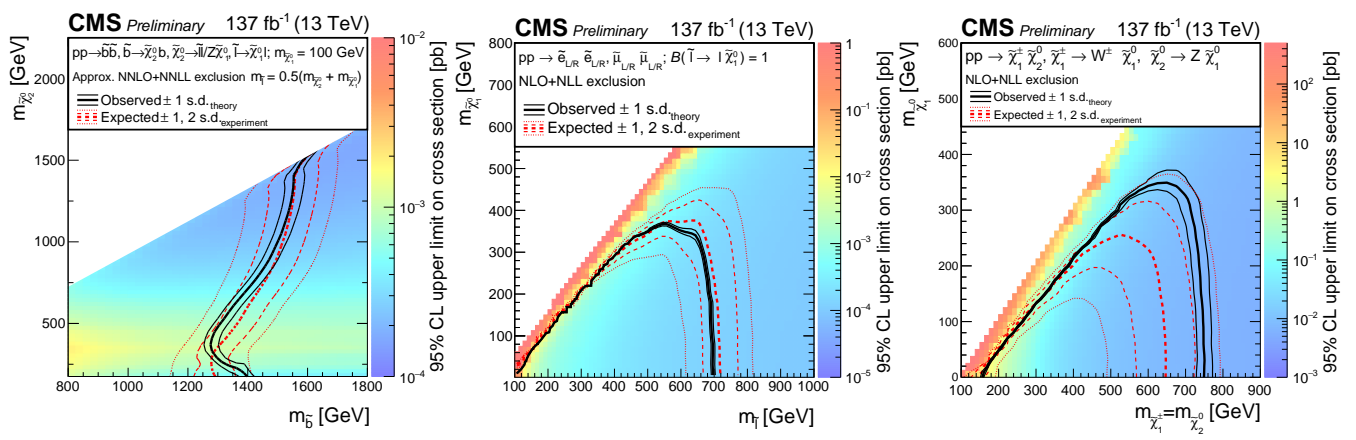

Figure 3: Cross-section upper limits and exclusion contours at 95\% CL for the bottom squark pair production (left), slepton pair production (middle), and $\widetilde{\chi}_{1}^{ \pm} \widetilde{\chi}_{2}^{0}$ production (right) [3].

squarks decay to multiple $W$ or $Z$ bosons, with at least one SS $W$ bosons pair. More interpretations in this regard can be founded in Ref. [8].

The $R$-parity conserving model of bottom squark production with subsequent decay of $\widetilde{b_{1}} \rightarrow$ $t \widetilde{\chi}_{1}^{ \pm}$yields a final state with two b quarks and four $W$ bosons as shown in Fig. 4 (left). In contrast, the $R$-parity violating model is that with pair-produced gluinos decaying to the lightest squark $(\widetilde{g} \rightarrow \widetilde{q} q)$, which decays to quarks and an off-shell $\widetilde{\chi}_{1}^{0}$. The $\widetilde{\chi}_{1}^{0}$ decays farther (violating the $R$-parity) into two quarks and charged lepton $\left(\widetilde{\chi}_{1}^{0} \rightarrow q \bar{q} \ell^{ \pm}\right)$as shown in Fig. 4 (right).

The baseline selection of this analysis requires at least two jets with $p_{T}>40 \mathrm{GeV}$ and two light SS leptons with $p_{T}>15 \mathrm{GeV}(10 \mathrm{GeV})$ for electrons (muons) and reject lepton pairs with invariant mass $\left(\mathrm{m}_{\ell \ell}\right)$ less than $12 \mathrm{GeV}$ to reject the Drell-Yan background. Events are then categorized based on the $p_{T}$ of the leptons forming the SS pair, the number of leptons ( 2 or more), and the value of the $p_{\mathrm{T}}^{\text {miss }}$. The SS lepton event categories are named: high-high $(\mathrm{HH})$ if both have $p_{T}>25 \mathrm{GeV}$, low-low if both have $p_{T}<25 \mathrm{GeV}$, and high-low (HL) otherwise.

The main backgrounds are considered to be from three sources. The first consists of events with two or more prompt leptons, including an SS pair. This background category includes a variety of low cross-section processes where multiple electroweak bosons are produced such as 

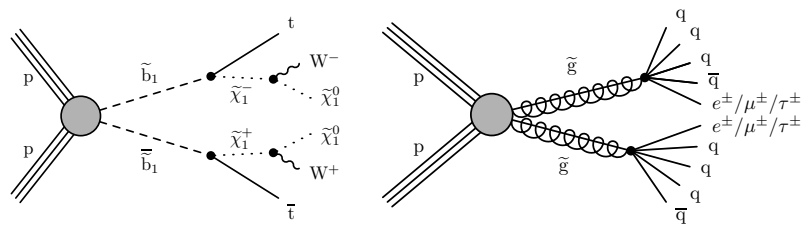

Figure 4: Diagrams of $R$-parity conserving models (left and middle) and P-parity conserving models in the SS dileptons analysis [8].
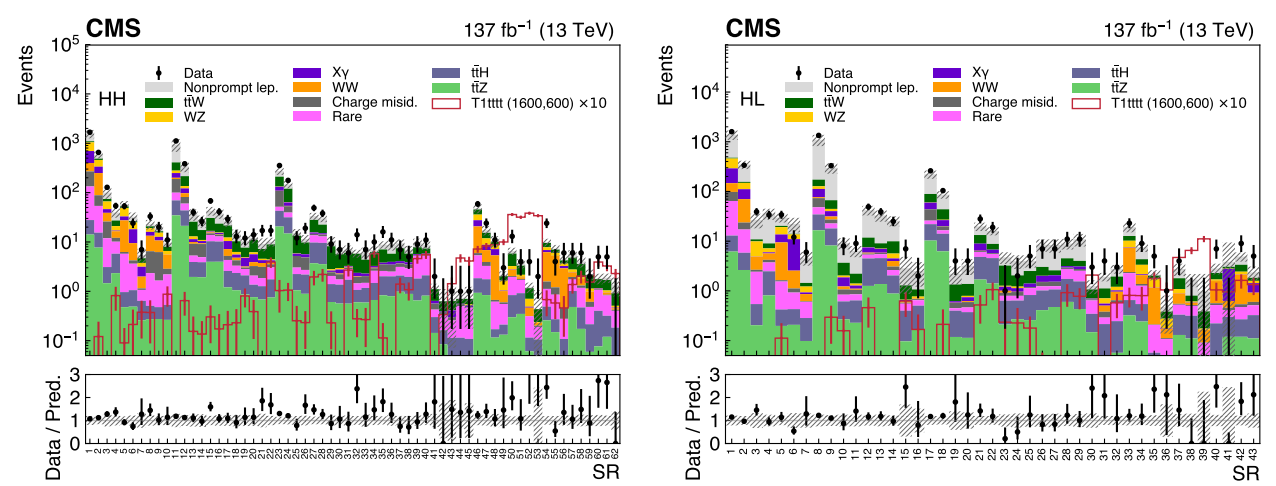

Figure 5: Expected and observed SR yields for the HH (left) and HL (right) signal categories. The hatched area represents the total statistical and systematic uncertainty in the background prediction [8].

$t \bar{t} W / Z / H, W Z$, and others. The shapes of these backgrounds are taken from simulations, while the normalizations are corrected in data CRs.

The second main background class is from events with two leptons one of them is nonprompt, either from a heavy flavor hadron decay or, more rarely, a misidentified hadron decay. This background class is estimated from data using the so-called "tight-to-loose" method, which accounts for the probability for a non-prompt to satisfy the tighter lepton selection $[9,10]$.

Finally, the third main background class is from events where the charge of a lepton is incorrectly measured due to electron bremsstrahlung. Using the number of OS pairs and the charge misidentification rate information, this background similarly estimated from data as the tight-toloose approach.

Background yields in two of the SRs are shown in Fig.5. Figure 5 shows that the observed data is in good agreement with the predicted background [8]. In all SR bins, the data is compatible with the background, except for a few bins where the agreement is subject to systemic uncertainty. The largest excess of events found by fitting the data with the background-only hypothesis is in HH bins 54 and 55, corresponding to a local significance of 2.6 and 1.8 s.d, respectively.

These results are then interpreted as experimental constraints on the cross-sections for the signal models. In the case of the $R$-parity violating models, upper limits at $95 \% \mathrm{CL}$ on the cross-section for gluino pair-production with each gluino decaying into four quarks and one lepton is shown in Fig. 6 (left). Gluino masses up to $2.1 \mathrm{TeV}$ are excluded. In the case of the $R$-parity conserving model, exclusion regions at $95 \% \mathrm{CL}$ in the plane of $m_{\widetilde{\chi}_{1}^{ \pm}}$versus $m_{\widetilde{b_{1}}}$ for the model with $R$-parity conserving decay chain is shown Fig. 6 (right). The $m_{\widetilde{\chi}_{1}^{0}}$ is set to $50 \mathrm{GeV}$. This model excludes $m_{\widetilde{\mathrm{b}_{1}}}$ up to $1000 \mathrm{GeV}$ [8]. 

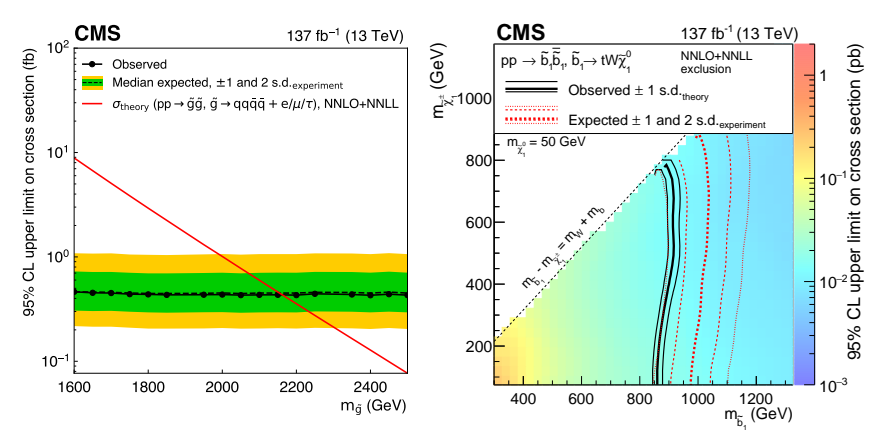

Figure 6: Upper limits at $95 \% \mathrm{CL}$ on the cross-section for $R$-parity violating gluino pair production with gluino decaying into four quarks and one lepton (left), and plane of $m_{\widetilde{\mathcal{X}}_{1}^{ \pm}}$versus $m_{\widetilde{\mathrm{b}_{1}}}$ for the model with $R$-parity conserving decay chain with $m_{\widetilde{\mathcal{X}}_{1}^{0}}$ is set to $50 \mathrm{GeV}$ (right) [8].

\section{Summary}

We presented two leptonic analyses looking for physics beyond the standard model in protonproton collisions at $13 \mathrm{TeV}$, corresponding to an integrated luminosity of $137 \mathrm{fb}^{-1}$. The first analysis targets final states with a same-sign light lepton pair or at least three charged light leptons. At least two additional jets are required in this analysis. The second analysis looks for an opposite-sign sameflavor pair of light leptons. This analysis targets final states with as well as without extra hadronic activity. The analyses are used to look for evidence of both electroweak and strong production of supersymmetry. The corresponding mass limits on supersymmetric particle production are estimated.

\section{References}

[1] S. Chatrchyan et al. [CMS], JINST 3 (2008), S08004 doi:10.1088/1748-0221/3/08/S08004

[2] I. Hinchliffe et al., Phys. Rev. D 55 (1997), 5520-5540 doi:10.1103/PhysRevD.55.5520

[3] The CMS Collaboration, CMS-PAS-SUS-20-001

[4] V. Khachatryan et al. [CMS], JHEP 04 (2015), 124 doi:10.1007/JHEP04(2015)124

[5] V. Khachatryan et al. [CMS], JHEP 12 (2016), 013 doi:10.1007/JHEP12(2016)013

[6] S. Chatrchyan et al. [CMS], Phys. Lett. B 718 (2013), 815-840 doi:10.1016/j.physletb.2012.11.036

[7] S. Chatrchyan et al. [CMS], JHEP 06 (2011), 026 doi:10.1007/JHEP06(2011)026

[8] A. M. Sirunyan et al. [CMS], Eur. Phys. J. C 80 (2020) no.8, 752 doi:10.1140/epjc/s10052020-8168-3

[9] A. M. Sirunyan et al. [CMS], Eur. Phys. J. C 77 (2017) no.9, 578 doi:10.1140/epjc/s10052$017-5079-z$

[10] A. M. Sirunyan et al. [CMS], JHEP 02 (2018), 067 doi:10.1007/JHEP02(2018)067 